\title{
FATORES ASSOCIADOS AO SURGIMENTO DA RESPIRAÇÃO BUCAL NOS PRIMEIROS MESES DO DESENVOLVIMENTO INFANTIL
}

\section{FACTORS ASSOCIATED WITH ONSET OF MOUTH BREATHING IN EARLY CHILD DEVELOPMENT}

\author{
Edson Theodoro dos Santos Neto ${ }^{1}$ \\ Rodrigo Walter Barbosa ${ }^{2}$ \\ Adauto Emmerich Oliveira ${ }^{3}$ \\ Eliana Zandonade ${ }^{4}$
}

Barbosa RW, Oliveira AE, Zandonade E. Fatores associados ao surgimento da respiração bucal nos primeiros meses do desenvolvimento infantil. Rev Bras Crescimento Desenvolv Hum. 2009; 19(2):237-248.

\section{Resumo:}

Objetivo: os problemas relacionados com a respiração bucal são vários e possuem diversas consequências na saúde humana. O estudo de coorte prospectiva se propõe a determinar os fatores de risco associados ao desenvolvimento da respiração bucal nos primeiros períodos do desenvolvimento infantil. Método: o grupo inicial constituiu-se por 86 bebês com idade de zero a três meses selecionados em áreas de abrangência do município de Vitória-ES. Realizaram-se sete visitas domiciliares, coletando-se dados sobre respiração bucal, mista, vedamento labial, alterações respiratórias intervisitas, estimulação da respiração nasal, ronco habitual ao dormir, uso de mamadeira, sucção de chupeta, sucção de dedo e aleitamento materno. A partir do grupo inicial, 67 crianças permaneceram em acompanhamento até a idade média de 29,42 meses ( \pm 2,49dp). Resultados: a prevalência de respiração bucal foi de 3,0\%. O aleitamento materno (ORaj $=0,27: 0,09-0,83)$ e a estimulação da respiração nasal (ORaj = 0,09:0,01 - 0,52) funcionam como fatores de proteção ao vedamento labial, enquanto a alteração respiratória intervisita (ORaj = 7,61: 1,09 - 53,01), sucção de chupeta (ORaj = 5,54 : 2,00 - 15,37) e o ronco (ORaj = 10,74:2,32 - 49,64) funcionam com fatores de risco. Conclusão: a gênese da respiração bucal inicia-se pela perda do vedamento labial, como conseqüência da interação de fatores positivos e negativos sobre o complexo neurológico, ósseo e muscular.

Palavras-chave: respiração bucal; hábitos deletérios; vedamento labial; aleitamento materno.

\footnotetext{
Doutorando em Epidemiologia em Saúde Pública pela Escola Nacional de Saúde Pública Sérgio Arouca/Fundação Oswaldo Cruz. Mestrando em Saúde Coletiva pela Universidade Federal do Espírito Santo.

Docente do Programa de Pós-Graduação em Saúde Coletiva da Universidade Federal do Espírito Santo.

Docente do Programa de Pós-Graduação em Saúde Coletiva da Universidade Federal do Espírito Santo.

Departamento: Trabalho desenvolvido no Departamento de Medicina Social da Universidade Federal do Espírito Santo. Responsável pela correspondência: Edson Theodoro dos Santos Neto

Endereço: Rua Guilherme Bassine, nº 97, São Pedro I, Vitória-ES, Brasil. - CEP: 29030-015. e-mail: edsontheodoro@uol.com.br Fontes de financiamento à pesquisa: CNPq, FACITEC, CAPES
} 


\begin{abstract}
:
Purpose: there are several problems related to mouth breathing and there is many consequences in the human health. In this context, this prospective cohort study aims to determine the risk factors associated to the development of mouth breathing in the first periods of children's development. Methods: The initial group was constituted of 86 babies aging from zero to three months old, selected in the encircled areas of Vitória district, state of Espírito Santo. Seven domiciliary visits were made, collecting data about oral breathing, mixed, labial closing, inter visits respiration alterations, nasal breathing stimulation, common snore when sleeping, the use of baby's milk bottle, pacifier suction, finger suction and breastfeeding. From the initial group, 67 children were assisted until the average age of 29,42 months old ( $\pm 2,49 \mathrm{dp})$. Results: The prevalence of mouth breathing was of 3,0\%. Breastfeeding (ORadj=0,27:0,09-0,83) and the nasal breathing stimulation (ORadj=0,09:0,01-0,52) worked as protection factors against labial closing, while the inter visit breathing alteration (ORadj=7,61:1,09-53,01), pacifier suction (ORadj=5,54:2,00-15,37) and snoring (ORadj=10,74:2,32-49,64) worked as risk factors. Conclusions: The genesis of the oral breathing initiates by the loss of lip closing, as consequence of the interaction of positive and negative factors about the neurological, bone and muscular complex.
\end{abstract}

Key words: oral breathing; deleterious habits; lip closing; breastfeeding.

\section{INTRODUÇÃO}

Desde o nascimento, a respiração surge como um reflexo neuromuscular congênito não condicionado imprescindível à manutenção da vida. A partir do crescimento e desenvolvimento do recém-nascido, reflexos condicionados, desejáveis ou não, mediados pela influência do ambiente, vão determinar o estabelecimento do padrão respiratório infantil. ${ }^{1}$

No fisiologismo respiratório, a passagem do ar pelas fossas nasais é o mecanismo que possibilita a funcionalidade natural do sistema respiratório, enquanto a passagem do ar pela boca faz com que o caráter oclusivo labial se altere, determinando o padrão respiratório bucal como um reflexo condicionado indesejável. ${ }^{2}$ Quando a mudança da fisiologia respiratória torna a respiração bucal uma atividade habitual crônica, alterações patológicas podem desencadear-se durante o ciclo da vida, refletindo-se em diversas áreas da saúde humana.
Os respiradores bucais possuem diminuição da elasticidade pulmonar ou da caixa torácica, ocorrendo redução do volume de gás que pode ser mobilizado a cada respiração. Conseqüentemente, ocorre diminuição do volume de ar exalado de forma forçada após esforço respiratório máximo. ${ }^{3}$ Essas características podem ser atribuídas às modificações no comportamento das curvaturas da coluna vertebral e do músculo diafragma.

Ao comparar respiradores bucais e nasais, Yi et $\mathrm{al}^{4}$ observaram diferenças significantes entre os grupos e evidenciaram que o grupo dos respiradores bucais apresentou diminuição da lordose cervical, aumento da cifose torácica, aumento da lordose lombar e anteversão da posição da pelve e menor distância excursionada pelo músculo diafragma do lado direito e esquerdo.

Quanto aos distúrbios craniofaciais, a falta de compressão da língua no palato influencia o desenvolvimento ósseo e muscular 
do sistema estomatognático e facial. Isso faz o palato duro tornar-se ogival enquanto a arcada dentária superior tende a deslocar-se para a frente e para a medial provocando distooclusão e mordida cruzada ${ }^{5}$. Essas alterações são responsáveis pelo aspecto prolongado e estreito da face, característico dos respiradores bucais. ${ }^{6}$

Além disso, os estudos que utilizam o exame radiográfico cefalométrico, como instrumento de investigação, constatam o aumento significativo na inclinação do plano mandibular e alteração nas proporções faciais anterior, posterior, superior e inferior nos respiradores bucais, indiciando a constituição óssea do perfil facial longo. ${ }^{7,8}$

No complexo bucodentário, as conseqüências da respiração bucal se verificam tanto na modificação na forma dos arcos e disposição dos dentes quanto nas doenças mais comuns da cavidade bucal. O hábito da respiração bucal associa-se à redução do tamanho dos arcos superior e inferior ${ }^{9}$, à maior ocorrência de oclusopatias, como mordida aberta ${ }^{6}$, e ao maior percentual de cáries dentárias e sinais de gengivite. ${ }^{10}$

Já no âmbito da fonética articulatória, o mau posicionamento lingual, desenvolvido em resposta à condição respiratória, afeta a produção dos fonemas fricativos (/s/ e /z/), chamados sigmatismos anteriores, e os padrões de deglutição. De acordo com Marchesan ${ }^{11}$, a alteração funcional implicada no hábito de respiração bucal é um dos maiores determinantes sobre a posição da língua na cavidade oral. $\mathrm{O}$ bloqueio aéreo faz com que a língua tome uma posição mais para anterior e para baixo, como uma forma compensatória, garantindo o processo da respiração. Por isso, verifica-se maior freqüência de deglutição atípica e dentalização de consoantes linguoalveolares ${ }^{12}$, alta prevalência de sigmatismo anterior ${ }^{13}$, de deglutição e fonação atípicas ${ }^{6}$ em respiradores bucais.

Os problemas relacionados com a respiração bucal são vários e são conhecidas diversas conseqüências na saúde humana, contudo as causas primárias da respiração bucal são pouco esclarecidas. Nesse contexto, este estudo se propõe a determinar os fatores de risco associados ao desenvolvimento da respiração bucal nos primeiros períodos do desenvolvimento infantil.

\section{MÉTODO}

Foi realizado um estudo longitudinal que acompanhou, durante 30 meses, a prevalência do vedamento labial em crianças com idade inferior a três meses, nascidas em áreas com baixo nível socioeconômico do município de Vitória, Espírito Santo. Foram escolhidas três áreas que apresentavam indicadores de mortalidade infantil da cidade de Vitória, que teve índice geral de 13,5/1000 nascidos vivos em $2001^{14}$.

O protocolo de pesquisa foi aprovado pelo Comitê de Ética em Pesquisa (CEP) do Centro de Ciências da Saúde da Universidade Federal do Espírito Santo, com registro $n^{\circ}$ 0020/2003. O Termo de Consentimento Livre e Esclarecido foi apresentado e obtido dos pais para inclusão na pesquisa.

Para o cálculo do tamanho da amostra, utilizou-se a população de nascidos vivos no município de Vitória em 2001, estimada em 4.521 crianças (segundo o site www.datasus.gov.br), e a prevalência de vedamento labial de 79,6\% num grupo de crianças normais. ${ }^{6}$ Considerouse a precisão desejada de $10 \%$ e o nível de significância de 5\%. O tamanho da amostra encontrado foi de 62 crianças. Portanto, a amostra de 86 crianças incluídas no estudo indica uma margem de segurança de $28 \%$ para considerar as possíveis perdas de seguimento.

Foram incluídas no grupo todas as crianças encaminhadas pelos agentes comunitários de saúde, com idade inferior a três meses, visitadas no período de novembro de 2003 a maio de 2004, quando o número previsto de crianças foi atingido. As crianças deviam residir em 
uma das três áreas de abrangência do estudo e estar matriculadas em uma das três unidades de saúde da família (USF) correspondentes.

Durante esse período, quatro pesquisadores, em duplas, iam até as unidades de saúde e acompanhavam a visita domiciliar do agente comunitário de saúde nas residências dos sujeitos de interesse. Nas visitas domiciliares, as mães são habitualmente orientadas com relação aos cuidados com o recém-nascido, relativos à higiene bucal, à respiração bucal, a hábitos de sucção nutritivos e não nutritivos, à importância da amamentação exclusiva por, no mínimo, seis meses para o desenvolvimento craniofacial. Também são mostradas as vantagens nutricionais, imunológicas e afetivas decorrentes da amamentação, bem como as práticas e posições mais favoráveis.

Na visita inicial, as mães foram convidadas a participar da pesquisa, recebendo esclarecimentos quanto à natureza do estudo e, sem exceção, aceitaram participar. Elas assinaram o Termo de Consentimento e, a seguir, foram realizados entrevistas e exames padronizados, com o registro dos dados em formulário.

As visitas subseqüentes ocorreram numa periodicidade programada (inicialmente de 3 em 3 meses depois de 6 em 6 meses) e serviram de controle observacional sobre o cumprimento das orientações passadas na primeira visita, quando foram detectadas as condutas das mães com o bebê. A cada visita, fazia-se o preenchimento de um novo formulário pela observação e entrevista estruturada. A coleta de dados iniciou-se em novembro de 2003 e encerrou-se em junho de 2006, totalizando sete visitas domiciliares.

\section{Definição das variáveis}

A variável aleitamento materno foi definida segundo o conceito da Organização Mundial de Saúde ${ }^{15}$, quando a mãe ou o responsável afirmava que a criança recebia leite materno diretamente do seio ou extraído, independen- temente de estar recebendo alimento sólido ou líquido.

As variáveis de hábitos de sucção nutritivos e não nutritivos (sucção de dedo, tempo de sucção de chupeta e uso de mamadeira) foram definidas perguntando-se, em cada visita, se a criança manifestava tal hábito. Em caso afirmativo, registrava-se o período quando a criança iniciou e, caso tivesse parado, quando parou.

Na visita inicial, as mães foram orientadas a ficar atentas para o início da perda do vedamento labial da criança. Assim que o percebessem, deveriam estimular na criança o fechamento da boca durante o sono e a desobstrução nasal com jatos de soro fisiológico, quando a criança estivesse com as narinas obstruídas por secreções.

A variável alteração respiratória intervisita foi coletada a partir da segunda visita. Se a mãe relatasse que a criança desenvolveu qualquer alteração nas vias aéreas inferiores ou superiores, registrava-se a alteração positiva. Já a variável estimulação da respiração nasal era definida se, mediante alguma alteração respiratória, a mãe respondesse que praticou no período intervisita as recomendações fornecidas. O ronco ao dormir foi registrado se a mãe escutasse ruídos respiratórios durante o sono da criança, fosse durante o dia, fosse durante a noite.

O vedamento labial foi determinado por análise observacional e por palpação do músculo mentoniano. Se, durante a visita, a criança mantivesse os lábios contatados e permanecesse com a boca fechada sem contração contínua do músculo mentoniano, registravase a presença do vedamento labial.

Para o exame de respiração bucal, os examinadores utilizaram espelhos anatômicos, semelhantes ao Espelho de Altmann, posicionados na entrada do espaço aeronasal da criança, para observar a expiração do ar. A criança que não conseguisse marcar com ar o espelho, apresentasse algum tipo de dificulda- 
de respiratória e/ou obstrução nasal e ausência de vedamento labial era considerada respirador bucal. Caso ela apresentasse passagem aérea nasal livre e ausência de vedamento labial, era considerada respirador bucal misto.

\section{Análise estatística}

A análise estatística foi realizada no programa SPSS for Windows 11.5, selecionando-se, em cada visita, o vedamento labial como variável dependente e o aleitamento materno, a sucção de dedo, a sucção de chupeta, o uso de mamadeira, a estimulação da respiração nasal, a alteração respiratória intervisita, a obstrução nasal intervisita e o ronco durante o sono da criança, como variáveis independentes. O nível de significância adotado para todos os testes de Qui-quadrado realizados foi de 5\%. Nos modelos de regressão logística, as variáveis que apresentaram o p-valor menor ou igual a 0,10 na análise univariada foram submetidas ao método Stepwise forward LR não condicional para seleção automática de variáveis. Esse método testa a entrada das variáveis com significância estatística por um escore estatístico e remove as variáveis utilizando a probabilidade da função de razão de verossimilhan- ça, baseada na estimativa de máxima verossimilhança parcial. Adotou-se o nível de significância de 0,10 para entrada e 0,20 para a saída das variáveis no modelo.

\section{RESULTADOS}

A Figura 1 mostra a prevalência dos hábitos de sucção nutritivos e não nutritivos e aleitamento materno de acordo com as visitas domiciliares. $\mathrm{O}$ aleitamento materno inicia-se com uma prevalência alta, acima de $90 \%$, e cai, gradativamente, próximo de $10 \%$, na sétima visita. A curva da prevalência do uso de mamadeira aproxima-se de uma parábola, inicialmente, com uma prevalência próxima a $40 \%$, na terceira visita, próxima a 70\%, e na sétima visita, em torno de 50\%. O hábito da sucção de chupeta permanece com uma prevalência entre $30 \%$ e $50 \%$ da primeira até a sexta visita, ficando abaixo de $30 \%$ somente na sétima visita. A prevalência do hábito de sucção de dedo aparece bastante elevada nas primeiras três visitas, reduz-se rapidamente na quarta visita e mantém-se próxima a zero até a sétima visita.

Figura 1. Apresentação das prevalências dos hábitos de sucção nutritivos e não nutritivos nas visitas domiciliares, Vitória-ES. 2008

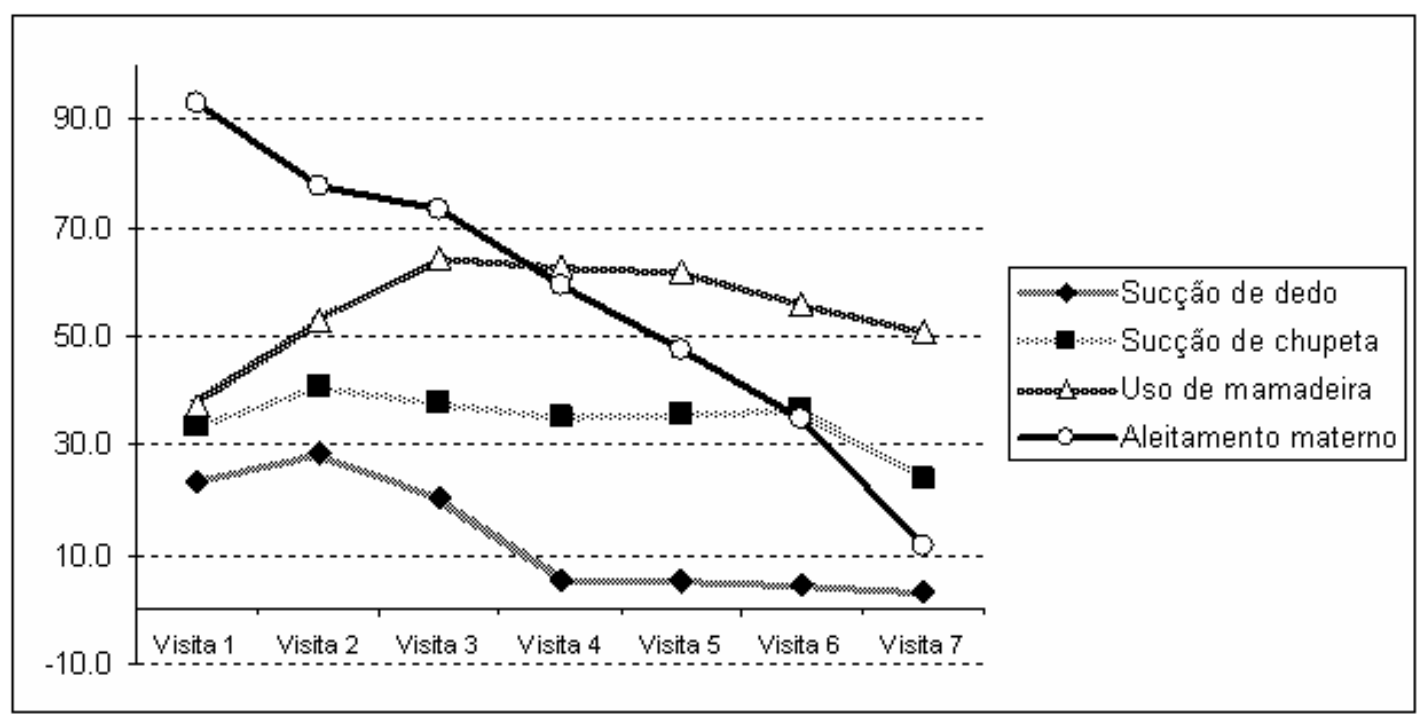


Na Figura 2, são mostradas as prevalências do vedamento labial da respiração mista e da respiração bucal. Nota-se que até a quarta visi- ta a respiração bucal inexiste, concomitantemente ocorre em certo grau a diminuição gradativa da presença de vedamento labial e o

Figura 2. Apresentação das prevalências do vedamento labial, da respiração mista e bucal nas visitas domiciliares, Vitória-ES. 2008

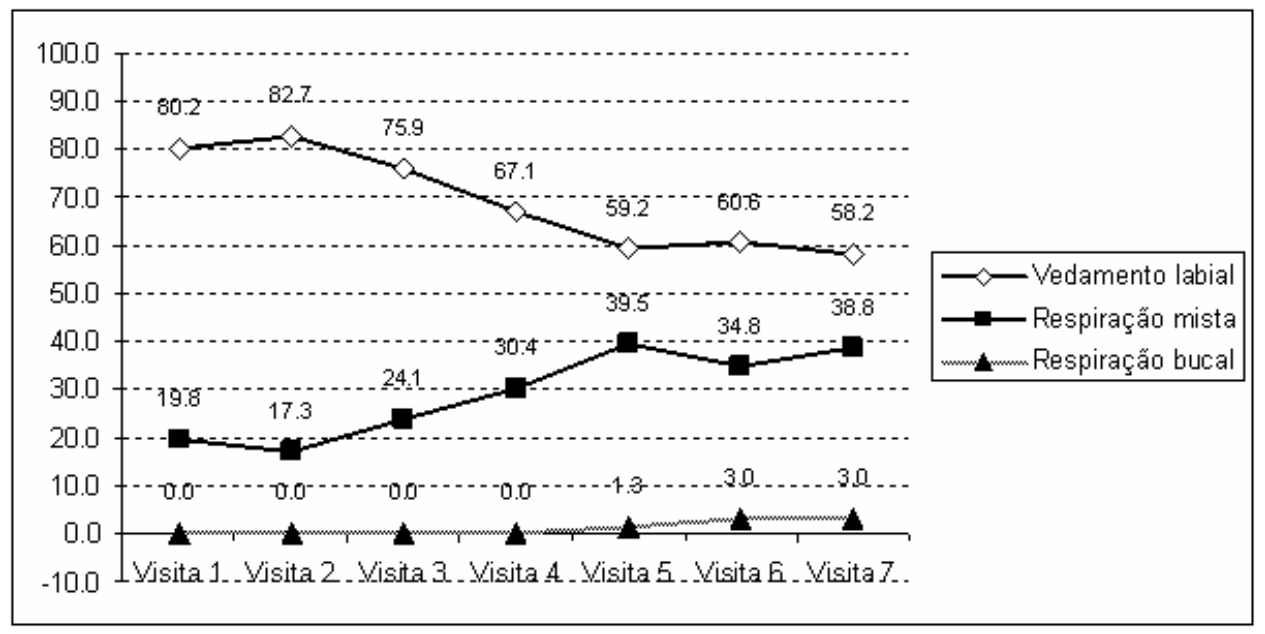

aumento considerável da respiração mista. Nesse cenário, o hábito da respiração bucal começa a instalar-se, sendo detectada sua presença a partir da quinta visita, permanecendo com uma prevalência constante na sexta e sétima visitas.

A Tabela 1 resume as análises univariadas e multivariadas, mostrando os resultados dos testes de qui-quadrado e da regressão logística, que correspondem, respectivamente, aos valores de odds ratio simples e odds ratio ajustados.

Na primeira visita (idade da criança 1,58 meses $+1,05 \mathrm{dp}$ ), apenas o uso de mamadeira aparece como uma variável de proteção à presença do vedamento labial. Na segunda visita (idade da criança 4,89 meses $+1,55 \mathrm{dp}$ ), a estimulação da respiração nasal funcionou como o único fator de proteção à presença do vedamento labial.

Na terceira visita (idade da criança 7,39 meses + 1,56 dp), o aleitamento materno e a estimulação da respiração nasal surgem como fatores de proteção enquanto o uso de chupeta aparece como um fator de risco para o vedamento labial. Entretanto, somente a estimulação da respiração nasal se estabelece como um fator de proteção à variável dependente no modelo de regressão logística.

Na quarta visita (idade da criança 11,61 meses + 2,16 dp), o aleitamento materno constitui-se num fator de proteção, enquanto a alteração respiratória intervisita e o ronco ao dormir destacam-se como fatores de risco à ausência do vedamento labial. Isso ocorre tanto na análise univariada quanto na multivariada.

Na quinta visita (idade da criança 16,39 meses $+2,88 \mathrm{dp}$ ), o aleitamento materno permanece como um fator de proteção, a sucção de chupeta reaparece como um fator de risco e o uso de mamadeira que, inicialmente, era um fator de proteção, torna-se fator de risco. Contudo, somente a sucção de chupeta aparece na análise final como um fator de risco significativo.

Na sexta visita (idade da criança 21,92 meses + 3,03 dp) e na sétima visita (idade da criança 29,42 meses + 2,49 dp), somente uma variável destaca-se como um fator de risco para a 
Tabela 1: Apresentação das variáveis significantes na Análise Bivariada de Qui-quadrado e no Modelo de Regressão Logistica.

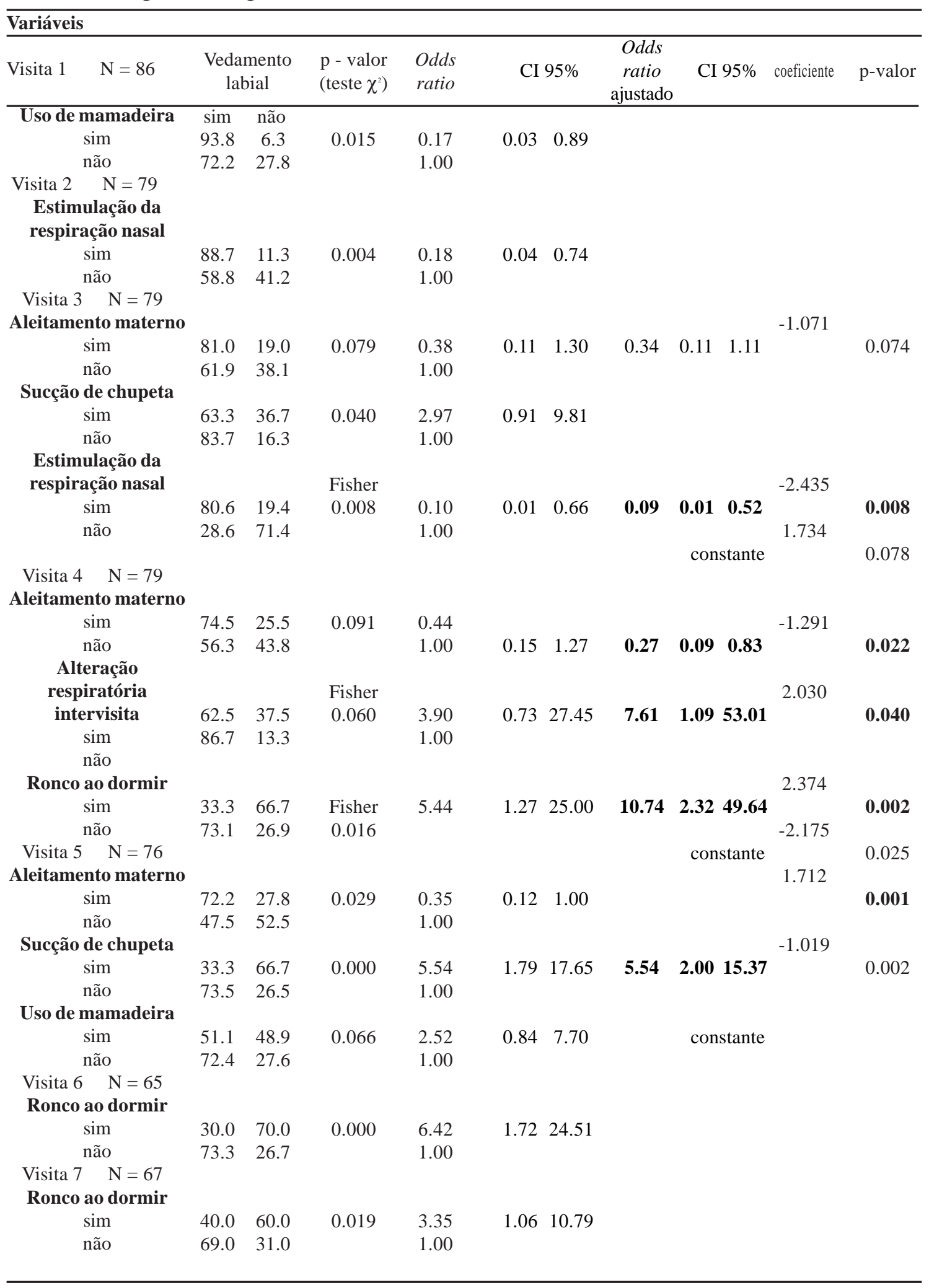


presença do vedamento labial, o ronco ao dormir.

\section{DISCUSSÃO}

Os resultados do estudo destacam os primeiros 16 meses de vida como cruciais para o desenvolvimento do padrão respiratório da criança, sendo o desfecho vedamento labial relacionado com diferentes fatores que possuem sua importância de atuação em momentos específicos da vida da criança.

Esse desfecho é considerado como o fator sintomático inicial do desenvolvimento da respiração bucal, pois estudos eletromiográficos em crianças respiradoras bucais e nasais ${ }^{16}$ observaram que, nos testes de repouso sem contato labial, no de repouso com contato labial e no de manutenção do esforço, as crianças respiradoras bucais apresentaram maior atividade muscular, sugerindo maior esforço para manter a postura labial. Isso proporciona aos indivíduos a abertura labial habitual.

A baixa prevalência final de respiração bucal $(3,0 \%)$ concorda com os achados de Vig ${ }^{17}$ que considerou a obstrução nasal total um achado clínico raro. O fato mais comum é a combinação simultânea de respiração nasal e bucal, ou seja, respiração mista. Isso não pressupõe que a respiração mista seja uma condição benéfica, contudo parece ser menos grave que a respiração bucal restrita.

O surgimento dos primeiros casos de respiração bucal da população acompanhada na Visita 5 coincide com a diminuição da prevalência de vedamento labial de $80,2 \%$ para $59,2 \%$, queda da taxa de aleitamento materno de $93,0 \%$ para $47,4 \%$, aumento da prevalência de uso de mamadeira de $37,2 \%$ para $61,8 \%$ e manutenção de altas taxas de sucção de chupeta, acima de $30,0 \%$, desde a Visita 1 . Sugere-se, então, que tais fatores estão, de alguma forma, associados ao aparecimento do reflexo da respiração bucal.
Por volta dos cinco meses, o aleitamento materno mostra-se como um fator de proteção importante para a prevenção da perda do vedamento labial, mantendo-se até em torno dos 16 meses. Contudo, é por volta dos 12 meses que o aleitamento materno é capaz de atingir o seu auge na proteção da perda do vedamento labial.

A explicação fisiológica da amamentação defende que, durante esse ato, o mamilo do seio materno sela completamente a boca da criança, impedindo a entrada de ar pela boca e forçando a passagem através do espaço aeronasal. ${ }^{18}$ Então, o ato da amamentação possibilita o aumento da força de vedamento labial, prevenindo o reflexo condicionado indesejável da respiração bucal. ${ }^{19}$ Além disso, proteção imunológica e nutricional proveniente do leite materno funcionaria como um fator de prevenção às infecções respiratórias ${ }^{20}$, que podem tornar-se cada vez mais freqüentes ao longo da vida da criança, fazendo com que crises de obstrução nasal forcem esse bebê a respirar pela boca até que haja perda do vedamento labial em repouso e a instalação definitiva da respiração bucal.

É importante destacar que, mesmo considerando as alterações respiratórias de modo generalizado, essas alterações exercem seu papel preponderante para a perda de vedamento labial em torno do $8^{\circ}$ ao $12^{\circ}$ mês de vida, pois a Visita 4 é o resultado de um histórico de alterações respiratórias desde a Visita 3. O detalhamento sobre quais alterações respiratórias influenciariam a perda de vedamento e a respiração bucal mereceria uma investigação à parte. Supõe-se que, independentemente da patologia que cause obstrução das vias aéreas superiores, o impedimento temporário da passagem aérea nasal freqüente obriga a passagem do ar pela boca.

O uso de mamadeira que apareceu como um fator de proteção inicial para o vedamento labial mostrou o seu efeito nocivo em torno dos 16 meses como um fator de risco. Essa altera- 
ção radical pode ser explicada principalmente pelas altas taxas de aleitamento materno até a Visita 4 que não permitiam o aparecimento do uso da mamadeira como um fator de risco importante. Comparando crianças amamentadas que utilizavam copo e mamadeira, Carrascoza et $\mathrm{al}^{21}$ concluíram que o uso de mamadeira, mesmo entre crianças que receberam aleitamento materno, interfere negativamente sobre o desenvolvimento orofacial, no que se refere à perda do selamento labial, repouso da língua no arco superior e formato maxilar anormal.

Em adição, a sucção de chupeta como um hábito de sucção não nutritivo tem revelado seus efeitos deletérios sobre diversos aspectos na saúde da criança. Estudos demonstram que o uso de chupeta interfere diretamente na duração do aleitamento materno, devido a uma “confusão de bicos”, pois existem diferenças mecânicas e padrões de sucção diferenciados estabelecidos pela criança entre o bico do seio materno, a chupeta e o bico da mamadeira ${ }^{22}$. Esse fenômeno levaria as crianças a "optarem" pela sucção dos bicos artificiais em detrimento à sucção do bico do seio materno. Isso porque a chupeta produz no organismo uma saciedade sensorial proporcionada pelo estímulo à sucção, salivação e deglutição, saturando de informações aferentes o sistema funcional da alimentação e competindo com a amamentação. ${ }^{23}$

A sucção de chupeta surge como fator de risco para a perda do vedamento labial com o tempo prolongado de uso, em torno de oito meses, e, com o passar do tempo, assume-se como o mais importante fator de risco, por volta dos 16 meses. Segundo Praetzel ${ }^{24}$, o uso de chupeta pode levar uma criança a ser um respirador bucal habitual, devido à compensação postural adquirida pela musculatura facial e da língua e pela mandíbula (boca aberta, hipotonicidade da musculatura facial e da língua), necessária para manter tal objeto na boca. Isso configura alterações nos padrões fisiológicos e neuromusculares faciais que geram desequilíbrios musculares e funcionais, gradativamente adaptativos na face e no organismo como um todo, que podem desencadear distúrbios miofuncionais. Depois dos 16 meses, grande parte das crianças tende a abandonar o uso de chupeta, porém as conseqüências decorrentes dessa substituição terão impacto na qualidade de vida infantil, forçando-a a desenvolver reflexos condicionados não desejáveis que podem perdurar ao longo de toda a sua trajetória de vida.

A sucção de dedo não se apresentou nem como um fator de risco nem como fator de proteção. Embora seja alta sua prevalência nos primeiros oito meses, cai drasticamente a partir do primeiro ano de vida. Esse fenômeno pode ser parcialmente explicado pelo aspecto psicológico, considerando que a criança se encontra na fase oral e desenvolve seu mecanismo de prazer pela boca. ${ }^{25}$ Embora não apresentando riscos ao vedamento labial, a sucção de dedo deve ser combatida devido ao seu efeito prejudicial na oclusão dentária.

No que se refere à estimulação da respiração nasal realizada pelas mães, como uma tentativa de prevenir a instalação do hábito da passagem do ar pela cavidade oral, parece ter tido seu efeito protetor por volta dos cinco meses iniciais, sendo muito importante em torno do $8^{\circ}$ mês de vida. Entretanto, depois desses períodos, parece não exercer nenhum efeito sobre o vedamento labial. A tomada de medidas para a prevenção do desenvolvimento da respiração bucal é um campo ainda pouco explorado, visto que não foi encontrada referência na literatura. Portanto, há necessidade de entender as causais iniciais do surgimento da respiração bucal a fim de propor medidas eficazes de prevenção.

Quanto ao ronco ao dormir, já é considerado como o resultado de manifestações das alterações respiratórias. O ronco ou ruído ao dormir apresenta-se, pela primeira vez, como um importante fator de risco forte por volta dos 12 meses de vida e estabelece-se como o 
único fator de risco à perda de vedamento em torno dos 22 e 29 meses. O estudo de Petry et $a{ }^{26}$ sobre prevalência de distúrbios respiratórios do sono mostrou que o ronco habitual e a respiração bucal foram os distúrbios mais prevalentes na população de escolares de nove a quatorze anos e estiveram significativamente caracterizados como fatores de risco à sonolência diurna. Isso sugere que crianças com tais distúrbios possuem uma qualidade de sono deficiente, o que se reflete em outros aspectos da qualidade de vida e saúde do respirador bucal.

A partir da necessidade de esclarecimento sobre as bases do surgimento da respiração bucal, o estudo propõe a "Hipótese da Gênese da Respiração Bucal”:

1. A instalação da síndrome do respirador bucal não se inicia no momento em que há obstrução parcial ou total das vias aéreas superiores pelo desenvolvimento hipertrófico da mucosa nasal, mas ocorre desde o momento em que há perda do vedamento labial, como conseqüência da interação de fatores positivos e negativos sobre o complexo neurológico, ósseo e muscular.

2. Os hábitos de sucção nutritivos e não nutritivos e as alterações respiratórias ocorridas durante os primeiros meses de vida funcionariam como fatores negativos condicionantes ao vedamento labial, enquanto a amamentação e a estimulação da respiração nasal da criança funcionariam como fatores positivos condicionantes.

3. Quando os fatores negativos condicionantes predominam em momentos específicos do desenvolvimento infantil, determinam a perda do vedamento labial, em conseqüência à instalação da respiração mista, que ocasiona o desenvolvimento do ronco habitual respiratório e, enfim, o estabelecimento da respiração bucal com obstrução nasal.
4. Nesse processo dinâmico, à medida que a respiração bucal se instala definitivamente, desencadeiam-se alterações corporais fisiopatológicas morfo-funcionais que atingem todo o indivíduo, influenciando o crescimento e o desenvolvimento postural físico e craniofacial, além do mecanismo respiratório.

Portanto, considera-se infundada a afirmativa de que a hipertrofia da adenóide, o desvio de septo nasal, a hipertrofia da mucosa dos cornetos nasais, a atresia maxilar, o palato ogival, a mordida aberta anterior, a mordida cruzada posterior, o perfil facial longo, as modificações no comportamento das curvaturas da coluna vertebral e do músculo diafragma sejam as causas da respiração bucal. São consideradas, portanto, como consequiências da cronicidade da respiração bucal.

O estudo sobre a gênese da respiração bucal tentou esquadrinhar os fatores e os momentos em que tais fatores atuam de maneira mais expressiva precipitando a ocorrência da perda do vedamento labial. Entretanto, é prematuro afirmar que todas as crianças que desenvolvem a perda do vedamento labial desenvolverão, irreversivelmente, a respiração mista ou bucal. Não é claramente definido por que grande parte dos respiradores bucais mistos não se tornam respiradores bucais restritos, mas é patente que a respiração bucal surge a partir da perda do vedamento labial.

As limitações do estudo referem-se ao grande desconhecimento sobre que outros determinantes proximais, intermediários ou distais estariam também influenciando a ocorrência da gênese da respiração bucal. Fatores relacionados com as condições domiciliares, ocorrência e recorrência de doenças respiratórias e alérgicas, fatores genéticos, fatores sociais, fatores associados ao uso e dependência de drogas lícitas e ilícitas no ambiente domiciliar podem exercer o seu papel na gênese da respiração bucal.

Além disso, o acompanhamento das crianças por um período maior poderia estabelecer maiores evidências, principalmente em relação 
ao diagnóstico de atopia e/ou asma, geralmente estabelecido a partir dos 36 meses de vida.

$\mathrm{O}$ estudo aprofundado sobre os fatores predisponentes à gênese da respiração bucal possibilitaria a formulação de estratégias preventivas interceptoras capazes de impedir o estabelecimento de alterações no crescimento e desenvolvimento no indivíduo.

Assin, o surgimento da respiração bucal coincide com a diminuição do vedamento labial e do aleitamento materno e, também, com o aumento do uso de mamadeira e da sucção de chupeta.

Os fatores de proteção associados à manutenção do vedamento labial são o aleitamento

\section{REFERÊNCIAS}

1. Moyers RE, Carlson DS. Maturação da neuromusculatura orofacial. In: Enlow DH, Crescimento Facial. 3 ed. Rio de Janeiro: Artes Médicas; 1993, p. 260-71.

2. Torres R. Interfuncionalidad. In: Torres R, Biologia de la boca: Estructura y funcion. 1 ed. Buenos Aires: Panamericana; 1973, p.519-551.

3. Barbiero EF, Vanderlei LCM, Nascimento PC, Costa MM, EscalabriniNeto A. Influência do biofeedback respiratório associado ao padrão quiet breathing sobre a função pulmonar e hábitos de respiradores bucais funcionais. Revista Brasileira de Fisioterapia 2007; 11(5): 347-353.

4. Yi LC, Jardim JR, Inoue DP, Pignatari SSN. The relationship between excursion of the diaphragm and curvatures of the spinal column in mouth breathing children. J Pediatr (Rio J). 2008; 84(2):171-177.

5. Mocellin L. Alteração oclusal em respiradores bucais. J Bras Ortod Ortop Maxilar 1997; 2: 45-8.

6. Emmerich AO, Santos-Neto ET, Zandonade E, Matos AM, Silva BP, materno e a estimulação da respiração nasal, enquanto o uso de mamadeira, a alteração respiratória intervisita e o uso de chupeta são os principais fatores de risco para a perda do vedamento labial.

O ronco, ao dormir, foi o principal distúrbio respiratório associado a perda do vedamento labial, como conseqüência do reflexo da respiração bucal.

A gênese da respiração bucal inicia-se pela perda do vedamento labial, como conseqüência da interação de fatores positivos e negativos sobre o complexo neurológico, ósseo e muscular.

Moraes JR. Estudo sobre alterações craniofaciais em crianças atópicas e/ou asmáticas, Hospital Universitário Cassiano Antônio Moraes, Vitória-ES, Brasil. UFES Rev Odont 2008; 10(1):39-47.

7. Lessa FCR, Enoki C, Feres MFN, Valera FCP, Lima WTA, Matsumoto MAN. Influência do padrão respiratório na morfologia craniofacial. Rev Bras Otorrinolaringol 2005; 71(2): 156-60.

8. Bizetto MSP, Maruo H, Shimizu RH, Guariza-Filho O. Estudo cefalométrico comparativo entre crianças respiradoras bucais e nasais nos diferentes tipos faciais. R Dent Press Ortodon Ortop Facial 2004; 9(1): 79-87.

9. Aznar T, Galán AF, Marín I, Domínguez A. Dental arch diameters and relationships to oral habits. Angle Orthodontist 2006; 76 (3): 441-445.

10. Nascimento-Filho E, Mayer MPA, Pontes P, Pignatari ACC, Weckx LLM. Caries prevalence, levels of Mutans streptococci, and gingival and plaque indices in 3.0- to 5.0-year-old mouth breathing children. Caries Res 2004; 38 : 572-575. 
11. Marchesan IQ. Adapted or Atypical Thrusting?, Int J Orofac Myology 1999; 25:15-17.

12. Hanson ML, Cohen MS. Effects of form and function on swallowing and the developing dentition. Am J Orthod 1973; 64: 63-82.

13. Pinto MA, Viana MM, Bommarito S. Sigmatismo anterior: Relação com a mordida aberta e com a respiração bucal. Revista Odonto 2003; 11(22): 73-83.

14. Prefeitura de Vitória. Plano Municipal de Saúde. Vitória: Secretaria Municipal de Saúde. Vitória: Secretaria Municipal de Saúde de Vitória - CEDOC - Centro de Documentação; 2001.

15. WHO World Health Organization. World Health Organization's infant feeding recommendation. Bull World Health Organ 1995; 73:165-74.

16. Tomé MC, Marchiori SC. Estudo eletromiográfico dos músculos orbiculares superior e inferior da boca em crianças respiradoras nasais e bucais durante o repouso com e sem contato labial. J Bras Ortodo Ortop Facial 1998; 3: 59-66.

17. Vig KWL. Nasal obstruction and facial growth: the strength of evidence for clinical assumptions. Am J Orthod Dentofac Orthop 1998; 113: 603-11.

18. Neiva FCB, Cattoni DM, Ramos JLA, Issler H. Desmame precoce: implicações para o desenvolvimento motor-oral. J Pediatr (Rio J) 2003; 79:7-12.

19. Sakashita R, Kamegai T, Inoue N. Masseter muscle activity in bottle- feeding with the chewing bottle teat: evidence from electromyographs. Early Human Development. 1996; 45: 83-92.

20. Post CLA et al. Fatores prognósticos de letalidade hospitalar por diarréia ou pneumonia em menores de um ano de idade: estudo de caso e controle. Rev. Saúde Pública 1992; 26(6): 369-378.

21. Carrascoza KC, Possobon RF, Tomita LM, Moraes ABA. Consequences of bottle-feeding to the oral facial development of initially breastfed children J Pediatr (Rio J). 2006;82(5):395-7.

22. Neifert M, Lawrence RA, Seacat J. Nipple confusion: toward a formal definition. J Pediatr.1995; 126: s125s129.

23. Emmerich AO et al. Relação entre hábitos bucais, alterações oronasofaringianas e mal-oclusões em pré-escolares de Vitória, Espírito Santo, Brasil. Cad Saúde Pública 2004; 20 : 689-97.

24. Praetzel JR. Distúrbios Miofuncionais da Face: Um novo paradigma de atuação para a Odontopediatria. JBP - J. Bras. Odontoped. \& Odontol. do Bebê 2002; 1(4): 87- 94.

25. Akerman B. A criança e um de seus objetos substitutivos do amor: a chupeta. Ortodontia, São Paulo 1975; 8(1): 29-37.

26. Petry C, Pereira MU, Pitrez PMC, Jones MH,Stein RT. The prevalence of symptoms of sleep-disordered breathing in Brazilian schoolchildren J Pediatr (Rio J). 2008;84(2):123-129.

Recebido em: 20 de março de 2009. Modificado em: 22 de julho de 2009. Aceito em: 14 de agosto de 2009. 